\section{Cuando las hormigas corretean por el cerebro: retos y realidades de la psiquiatría cultural}

\author{
When ants crawl around the brain: \\ challenges and facts in cultural psychiatry
}

\begin{abstract}
1 Departamento de Antropología, Universidad Rovira i Virgili, Tarragona, España.

Correspondencia A. Martínez-Hernáez Departamento de Antropología, Universidad Rovira i Virgili. Plaza Imperial Tarraco $s / n$, 43005 Tarragona, España angel.martinez@urv.net
\end{abstract}

\begin{abstract}
Culture influences the experience, expression, course, evolution, prognosis, and treatment of mental disorders (as well as mental health policy) but has been neglected by general psychiatry, which has failed to integrate it appropriately into epidemiological research, clinical practice, and health policies. The main reason for this failure has been a lack of awareness concerning the "culture of psychiatry". This article aims to contribute to the theoretical debate in cultural psychiatry by reviewing studies on: (i) theoretical discussions and ethnographic studies of mental health services, (ii) cross-cultural comparative studies on psychiatric disorders, and (iii) the use of the cultural skills model with ethnic minorities and refugees.
\end{abstract}

Mental Health; Psychiatry; Culture
Ángel Martínez-Hernáez ${ }^{1}$

\section{Introducción}

El título de este artículo es una traducción adaptada de una expresión Igbo (Nigeria), utilizada por este grupo étnico para denotar el malestar somático característico de los trastornos mentales 1: "things like ants keep on creeping in various parts of my brain". Se trata de un idiom of distress o expresión de malestar que permite que el lector se ubique en la posición del clínico, epidemiólogo e investigador que debe trabajar con interlocutores ajenos a su cultura. ¿Qué significa esta frase? ¿A qué realidades de malestar y aflicción nos está remitiendo? ¿Podemos traducir esta expresión a otros lenguajes no sólo idiomáticos, sino también culturales? ¿ Nos habla de un tipo de experiencia que ya nos es conocida o nos enfrenta a formas de sufrimiento que sólo adquieren consistencia en un determinado mundo local?

Las preguntas que generan los idioms of distress han sido durante largo tiempo objeto de estudio de la psiquiatría transcultural, así como de la más contemporánea psiquiatría cultural. En términos descriptivos podemos decir que la primera ha sido a la investigación en territorios no-occidentales durante el periodo colonial y postcolonial, como la segunda al análisis de las minorías étnicas en contextos nacionales de multiculturalidad; aunque esta distinción no sea siempre operativa debido a las diferencias terminológicas según autores, escue- 
las y tradiciones nacionales 2 . Psiquiatría transcultural 3, etnopsiquiatría 4, antropología psiquiátrica 5 , psiquiatría comparada 6 , salud mental multicultural 7 y otros muchos términos, entre el que se cuenta el más moderno de psiquiatría cultural 8,9, conviven en lo que ha sido la historia de este campo fronterizo entre el estudio de los trastornos mentales y la indagación de las dinámicas de la cultura.

La psiquiatría transcultural aparece vinculada históricamente a la comparación de cuadros clínicos de diferentes culturas y al debate sobre la universalidad de las nosologías, como en los estudios comparativos de Kraepelin 10 sobre la dementia praecox o sus investigaciones sobre el koro, el amok y el latah; tres culture-bound syndromes del sudeste asiático que el clínico alemán entendió (hoy sabemos que de forma errónea) como expresiones étnicas de la depresión, la epilepsia y la histeria, respectivamente. También encontramos bajo este epígrafe los estudios comparados sobre las terapias tradicionales 11, así como viejas discusiones sobre el carácter normal o patológico de las conductas extáticas de los chamanes 12 , sobre el potencial del sueño 13 , sobre la eficacia curativa de los rituales 14 o sobre la universalidad o particularidad del Complejo de Edipo 15.

La psiquiatría cultural, por su parte, es un término que ha adquirido presencia en la literatura internacional sólo en fechas recientes, aunque haya acabado instituyéndose como concepto general para definir las investigaciones sobre la cultura, los tratamientos y los trastornos mentales. La razón es que los procesos de globalización 16 han modificado las condiciones de los estudios comparativos al convertir la diversidad cultural en un paisaje cotidiano en la mayoría de los contextos nacionales. La mayor dispersión por la desubicación de los grupos étnicos, el aumento de las migraciones transnacionales, la configuración multiétnica de las sociedades, el reto de la cohesión social y de las políticas multiculturales o las necesidades asistenciales de los refugiados de conflictos bélicos y genocidios son realidades y desafíos que han ofrecido protagonismo a una psiquiatría cultural que puede entenderse como la búsqueda de respuestas ante una sociedad en donde lo exótico se ha tornado ya cotidiano y lo cotidiano ha adquirido un carácter híbrido y mestizo.

En un artículo reciente, Kirmayer \& Minas 2 han apuntado que los tres grandes ámbitos de la psiquiatría cultural han sido: (1) los estudios comparativos de los trastornos psiquiátricos y las terapias tradicionales; (2) los esfuerzos en responder a las necesidades de poblaciones cul- turales diversas como los indígenas, los inmigrantes y los refugiados; y (3) el estudio etnográfico de la propia psiquiatría. Grosso modo, aquí entendemos que estas tres líneas han tendido a asociarse en la práctica y de forma respectiva con:

- La investigación epidemiológica y etnográfica de tipo comparativo de los trastornos mentales. Esta línea puede considerarse el eje central de la psiquiatría transcultural y la etnopsiquiatría clásicas;

- La asistencia psiquiátrica en contextos interculturales y el debate sobre las políticas públicas de salud mental. Esta orientación constituye el campo de la psiquiatría cultural entendida stricto sensu;

- El análisis de la psiquiatría como sistema cultural. Este campo aparece más vinculado a la antropología y sociología médicas, aunque también a la teoría psiquiátrica y a la historia de la psiquiatría.

Las tres orientaciones pueden parecer campos diversos en lo que respecta a los temas estudiados y las filiaciones disciplinares; sin embargo, en todos estos casos el elemento unificador ha sido la cultura como eje explicativo de la psicopatología y de su tratamiento. Por esta razón, aquí preferimos utilizar el término psiquiatría cultural como categoría comprehensiva que incluya las diferentes investigaciones y teorías sobre las relaciones entre la cultura, la terapia y los trastornos mentales. Un uso semejante puede encontrarse en el vasto trabajo de Tseng ${ }^{9}$ y en la mayoría de los autores contemporáneos 2,9,17.

Entendida en estos términos, la psiquiatría cultural ha mostrado un extraordinario crecimiento en los diferentes niveles apuntados, a la vez que un impacto muy limitado en la psiquiatría general. La postergada validación cultural del Diagnostic and Statistical Manual of Mental Disorders - Fourth Edition (DSM-IV) 18 es un buen ejemplo de esta exigua influencia, así como la desatención del tema multiétnico en muchas políticas públicas de salud mental, la escasa formación cultural de los profesionales y la ausencia de investigaciones que incluyan las relaciones - siempre complejas - entre biología y cultura a la hora de abordar los trastornos mentales. La psiquiatría cultural continúa siendo una "pariente pobre" de una psiquiatría general más orientada al estudio de los neurotransmisores que a la investigación de la influencia de las transformaciones culturales en la salud mental, más volcada en la psicofarmacología que en los contextos sociales de producción del sufrimiento, más encaminada a la tecnificación de los instrumentos diagnósticos 
que a la comprensión de los idioms of distress. Con todo, los enfermos mentales no son islas psicopatológicas carentes de contexto. Es tan incompleta una psiquiatría que no valore el contexto, como una psiquiatría que no dé cuenta de los procesos psíquicos y/o biológicos.

En este artículo vamos a revisar críticamente el campo de la psiquiatría cultural a partir de sus aportaciones a la teoría, la investigación comparativa y la asistencia en contextos multiculturales. Como tendremos ocasión de observar, en estos tres ámbitos la cultura se revela como una dimensión estratégica para la investigación de los trastornos mentales, así como para repensar la teoría psiquiátrica contemporánea.

\section{La teoría, o el descubrimiento de la cultura profesional}

La contribución teórica más destacada de la psiquiatría cultural no ha sido confirmar que la cultura es una variable importante en la determinación o co-determinación de la psicopatología, sino hacer consciente que la psiquiatría, como conjunto de saberes y de prácticas, es también un sistema cultural. Es lo que podemos llamar el "descubrimiento de la cultura profesional” que había permanecido invisibilizada por la existencia de una demarcación fuerte entre ciencia y cultura, entre sujeto y objeto de la investigación, entre profesional y paciente. Los primeros integrantes de estas relaciones representaban la racionalidad; los segundos el ámbito de la creencia.

La psiquiatría cultural - al menos en sus versiones más destacadas 19,20,21,22,23 - ha introducido la idea que las nosologías psiquiátricas son tentativas de otorgar nombres a los fenómenos psicopatológicos, pero también categorías dependientes de los contextos históricoculturales en donde han sido creadas. En oposición a la presunción baconiana que el lenguaje científico es el espejo de la naturaleza, ahora reaparece la evidencia que este lenguaje no es isomórfico con el orden de las cosas, sean éstas enfermedades orgánicas o trastornos mentales, pues siempre existe una distancia entre las categorías (ideacionales) y los procesos patológicos (reales). Esta afirmación ya era planteada con belleza literaria por Breuer 24 en las últimas páginas de Estudios sobre la Histeria, cuando parafraseando a Shakespeare decía "aún lo mejor en este género no son más que sombras”.

La concepción baconiana del lenguaje ha estado presente en los paradigmas hegemónicos en psiquiatría, especialmente en la corriente denominada neokraepelinismo, la cual a me- nudo ha llevado el ideal del determinismo biológico y del positivismo más allá que el propio Kraepelin 21. El manual diagnóstico de la Asociación Americana de Psiquiatría (DSM-IV 18 y DSM-IV-TR 25) y sus versiones inmediatamente anteriores (DSM-III 26 y DSM-IIIR 27) son buena prueba de esta posición, pues en ellos se parte de la ilusión de una clasificación ateórica, asocial y acultural de los trastornos mentales. En otro lugar 28 hemos analizado como esta presunción se basa en la posibilidad de un conocimiento científico que sea la "copia de los hechos" y como de aquí se deriva, a su vez, la asunción de un saber neutral y sin interferencias del mundo social, cultural y económico-político en donde ha sido producido.

Desde la psiquiatría cultural se entiende que los manuales neokraepelinianos son resultado de las epistemologías naturalistas de los trastornos mentales y concretamente del uso de la analogía botánica o more botánico que articuló la medicina de las especies de tipo neohipocrático, pero también las clasificaciones de Kraepelin. El problema, claro está, es que una taxonomía botánica difiere ontológicamente de una clasificación de trastornos psiquiátricos, pues mientras en la primera pueden establecerse correspondencias más ajustadas entre un objeto y una categoría, en la segunda el asunto es mucho más complejo. La razón es que las manifestaciones de los trastornos mentales son fundamentalmente realidades conductuales que requieren de un mayor grado de abstracción analítica. Un ejemplo nos lo ofrece el fenómeno de la comorbilidad y la dificultad para discernir en estos casos a qué trastorno pertenecerá una determinada manifestación: ¿̇a qué categoría pertenecerá la conducta agresiva del paciente, a su trastorno esquizofrénico, a su trastorno de personalidad o acaso a su trastorno por uso de substancias?

El uso de las categorías psiquiátricas como realidades más que como representaciones ha conducido al efecto definido por Kleinman como "falacia categorial" 19,29, especialmente cuando estamos exportando nosologías y criterios diagnósticos a contextos culturales en donde éstos no adquieren coherencia ni sentido, o adoptan otras coherencias y otros sentidos. Una de las consecuencias de la falacia categorial es la tendencia a forzar la universalidad de los criterios diagnósticos, cuando sería tan relevante, como oportuno, partir precisamente de la diversidad de síntomas y cuadros clínicos, como es el caso de los culture-bound síndromes, para desarrollar teorías que pudiesen dar cuenta de la variación. Otra de las derivaciones del problema es la confusión que se establece 
entre validez (validity) y fiabilidad (reliability) cuando la segunda, es decir, el grado de consenso y reproductibilidad de los juicios diagnósticos, es utilizada como criterio subrepticio para validar las nosologías.

Un símil de Kleinman 19 puede ser útil en este punto. Supongamos que diez psiquiatras estadounidenses que han sido entrenados en las mismas técnicas y criterios diagnósticos deben entrevistar a diez amerindios que se encuentran en la primera semana de duelo posterior a la muerte de su esposa. Los profesionales podrán determinar con un $90 \%$ de consistencia que siete de los pacientes escuchan la voz del espíritu de su esposa, el cual dialoga con ellos desde el más allá. En este caso estaremos ante un elevado grado de concordancia y fiabilidad. Ahora bien, la determinación de si esta experiencia es el síntoma de un estado mental anormal no podrá delegarse en la reproducibilidad de los juicios diagnósticos, sino en la interpretación de esta manifestación dentro de la cultura del sujeto. Como es sabido, en muchas culturas amerindias la experiencia de "oír voces" de la esposa o esposo fallecido es una forma pautada y consuetudinaria de vivir el duelo y no puede asociarse con un trastorno psicótico o depresivo, tal como ha reconocido el propio DSM-IV. Diagnosticar la experiencia auditiva de un amerindio en duelo como el indicio de un trastorno conducirá a un juicio con una elevada fiabilidad, pero a la vez con nula validez.

La validez de las nosologías no puede soslayarse con una apelación a la concordancia y reproducibilidad de los juicios diagnósticos, especialmente en el caso de los trastornos mentales, pues a la carencia de pruebas diagnósticas objetivas se suma el hecho que los síntomas psiquiátricos se superponen e imbrican con manifestaciones normales de la adversidad humana como la tristeza, la frustración, la infelicidad, el temor o el duelo, entre otras muchas. La conciencia de la relatividad cultural (a no confundir con el relativismo) de las nosologías, los juicios clínicos y los propios trastornos mentales es la garantía, en lugar del obstáculo, para el desarrollo de un conocimiento científico y racional. La crítica que puede realizarse a los presupuestos del neokraepelinismo - y del determinismo biológico por extensión - no es precisamente su exceso de cientificidad, sino su falta de ella a la hora de abordar una complejidad que no puede reducirse a determinaciones genéticas y a la ilusión de un mundo y unas taxonomías estables. El contexto social, cultural y económico-político de la aflicción es una realidad que está ahí, como los cro- mosomas y los neurotransmisores, mostrándose necesario para distinguir correctamente un caso de un no caso, para la tarea de interpretación clínica, para la puesta a prueba de las nosologías, para la traducción y adaptación de los instrumentos diagnósticos y epidemiológicos, para la determinación de la etiología, sintomatología, curso, evolución y pronóstico de muchos de los trastornos, para la dosificación adecuada de los psicofármacos, para la elaboración de políticas públicas y para la reflexión sobre la propia práctica profesional.

Las etnografías realizadas sobre los dispositivos psiquiátricos y las redes de asistencia en salud mental han abundado en esta misma dirección. Desde los trabajos institucionales clásicos sobre el modelo manicomial 30 , sabemos que las instituciones generan dinámicas de control social que no siempre responden al ideal terapéutico. El estudio de las interacciones sociales producidas en los centros de salud mental nos informa del peso de los intereses corporativos de los diferentes profesionales, de las estrategias de los pacientes y familiares, de las congruencias y disensiones entre los discursos teóricos y las prácticas y de la construcción cultural de la realidad clínica 22 . Los profesionales se convierten en este tipo de investigaciones en objeto de estudio y, por tanto, en sujetos culturales del mismo orden que pacientes y familiares. La etnografía se convierte en este punto en un instrumento reflexivo para repensar la "cultura de la psiquiatría”.

\section{La investigación comparativa, o el problema de la variabilidad}

Hay tres ámbitos que han captado de forma preferente la atención en las investigaciones comparativas. El primero de ellos es la cuestión de la variabilidad sintomatológica y su desafío a la constitución de una nosología universal. El segundo es el dilema de la diferencia en el pronóstico de enfermedades como la esquizofrenia entre los países desarrollados y los países en vías de desarrollo. El tercer ámbito - aunque no por ello menos importante - alude a los problemas en la traducción y adaptación cultural de los instrumentos diagnósticos y las escalas. Las tres líneas de investigación pueden resumirse en lo que podemos llamar "el problema de la variabilidad" y se han desarrollado en la estela - y también en la crítica - de los grandes proyectos comparativos, como el International Pilot Study of Schizophrenia (IPSS) 23, el Determinant of Outcome of Severe Mental Disorders (DOSMD) 31, el Collaborative Study on 
the Assessment of Depressive Disorders $32 \mathrm{o}$ el mucho más reciente World Mental Health Survey Initiative 33 .

La cuestión de la variabilidad sintomatológica y su desafío a la constitución de una nosología universal

La cuestión de la diversidad sintomatológica no es nueva en el conocimiento psiquiátrico. Ya Kraepelin 10 subrayó la presencia de una peculiaridad expresiva de los trastornos mentales en las culturas no occidentales. Los datos contemporáneos inciden en esta misma dirección, aunque sin replicar los resultados concretos de las excursiones etnopsiquiátricas del psiquiatra alemán. Por ejemplo, en el IPSS 23 se halló una mayor frecuencia de síntomas afectivos, ideas delirantes y delirios de inserción del pensamiento en los pacientes de las sociedades industrializadas, mientras en los contextos no industrializados "las voces que hablan al sujeto" y las alucinaciones visuales fueron más prevalentes. También se encontró una mayor proporción de pacientes del subtipo paranoide en Reino Unido, así como una mayor frecuencia de síntomas de desrealización en Estados Unidos en comparación con India, una mayor presencia de manía en Dinamarca y una alta tasa de esquizofrenia catatónica y del subtipo no especificado en India.

Los datos del IPSS tienen su continuidad en los resultados del DOSMD 31, donde se indica que los casos de esquizofrenia de tipo agudo son el doble en los países en vías de desarrollo frente a los países desarrollados y se afirma que mientras los casos de esquizofrenia catatónica en estos últimos son prácticamente inexistentes, en los países en vías de desarrollo alcanzan el $10 \%$. En cuanto al subtipo hebefrénico se indica que si bien se ha diagnosticado a un $13 \%$ en los países desarrollados, en los países en vías de desarrollo sólo se halla en un $4 \%$.

Las investigaciones sobre otros trastornos mentales han ofrecido resultados muy similares. En el ya clásico Collaborative Study on the Assessment of Depressive Disorders ${ }^{32}$, realizado en cinco países (Canadá, India, Irán, Japón y Suiza), se sostiene que mientras los sentimientos de culpa y auto-reproche aparecen en el $68 \%$ de la muestra suiza, sólo está presente en un $32 \%$ en la de Irán. Las ideas de suicidio también exhiben cierta diversidad, pues mientras su frecuencia es del 70\% en Canadá, no alcanza el 50\% en Irán. La agitación psicomotora adquiere, por su parte, una mayor presencia entre los pacientes de Teherán frente a la media de los diferentes centros y la somatización en- tre estos pacientes prácticamente dobla la frecuencia de los afectados de Canadá o de Suiza.

Como los propios autores de este estudio reconocen que existe una sobre-representación en la muestra de los pacientes occidentales y occidentalizados, se puede hipotetizar una mayor variabilidad que la aquí apuntada. En otros estudios transculturales sobre este trastorno se ha informado que en determinados contextos son raros el sentimiento de culpa, las ideas de suicidio, los sentimientos de desesperanza o la baja auto-estima. Contrariamente, en estas sociedades parecen ser comunes los síntomas somáticos y cuasi-somáticos, como los trastornos del sueño, del apetito, de la energía, de las sensaciones corporales y del funcionamiento motor 34 . La idea que los occidentales psicologizan y los no occidentales somatizan es ya un lugar común de los estudios comparativos, aunque esta información deba leerse sin maximalismos y de forma crítica 35 , pues también algunos estudios indican que en los contextos occidentales se expresa el ánimo deprimido mediante un lenguaje somático 36 . En realidad, uno de los problemas de las grandes investigaciones comparativas es su tendencia a esquematizar, constituyendo grandes bloques como "culturas occidentales" y "culturas nooccidentales” o "países desarrollados” y "países en desarrollo", que no son útiles para abordar las diferencias intrínsecas a cada uno de estos bloques y a cada uno de los contextos nacionales analizados; los cuales, con toda seguridad, muestran una gran variabilidad interna en términos de grupos étnicos, clase social, tendencia religiosa y/o mundo rural versus mundo urbano, por citar sólo algunas dimensiones.

Otra de las limitaciones es la falta de investigaciones que incluyan síntomas ajenos a los criterios diagnósticos preestablecidos. De ello se deriva que en muchas ocasiones se apunte como colofón que existe una uniformidad transcultural de los cuadros, cuando la homogeneidad ha sido forzada metodológicamente y la diversidad es evidente incluso con posterioridad a este proceso. Posiblemente ésta sea la razón de la reluctancia a incluir los culture-bound sindromes en este tipo de investigaciones.

La solución más frecuente al problema de la variabilidad ha consistido en la aplicación del modelo patogenia/patoplastia; una teoría introducida por Birnbaum (1878-1958) que Kraepelin utilizó para distinguir entre el núcleo patogénico duro (patogenia) y las condiciones circunstanciales que impone la procedencia étnica, las formas de vida y la biografía del paciente (patoplastia). Este modelo se aplica frecuentemente en la psiquiatría contemporánea, 
asociando la patogenia con la forma del síntoma y la patoplastia con el contenido. Leff 37 , por ejemplo, argumenta que mientras la forma comprende aquellas características esenciales que lo distinguen de otros síntomas diferentes, el contenido puede ser común a una variedad de manifestaciones y se deriva del contexto cultural del paciente. Un ejemplo puede ser oportuno en este punto: los pacientes depresivos severos pueden oír voces diciéndoles que son criminales o pecadores. Los pacientes creyentes tendrán más tendencia a ser pecadores y los no-creyentes a ser criminales. La forma o patogenia será para Leff el hecho que en ambas situaciones estamos ante alucinaciones auditivas. El contenido o patoplastia, por su parte, adquirirá un carácter más volátil y dependiente de la cultura del sujeto, por ejemplo: sus creencias religiosas.

El modelo patogenia/patoplastia ha sido utilizado de forma generalizada en las investigaciones comparativas por su capacidad para establecer un equilibrio, aunque a nuestro juicio precario, entre la universalidad de la psicopatología y la diversidad de los síntomas 28 . Mientras se respeta una evidente variabilidad, se hace énfasis en que la forma (o significante) es lo verdaderamente importante, pues es la que permite inscribir los síntomas en un marco nosológico determinado. El contenido, por su parte, es observado como una realidad afectada por las culturas locales, pero a la vez secundaria para el discernimiento de los cuadros. Ahora bien, la fórmula en cuestión no ofrece una solución para una parte importante de los casos.

Una de las carencias del modelo patogenia/patoplastia es que no permite contextualizar de manera adecuada. En la medida que lo patoplástico es del orden de la anécdota, no es determinante para definir, por ejemplo, si "oír voces" es una manifestación psicótica o una forma consuetudinaria de vivir el duelo entre ciertos grupos culturales, como los amerindios. Otra de las limitaciones es que en determinadas situaciones la hegemonía de la forma sobre el contenido se invierte. Una sencilla pregunta puede ilustrar esta carencia: ¿La diferente manifestación de la depresión en síntomas de somatización o de psicologización qué es, una diferencia de forma o de contenido? Parecería que lo variable en este caso sería la forma, mientras que el contenido, llamémosle "ánimo deprimido", sería lo invariable. ¿Cuál será en este caso el criterio para definir lo patogénico y lo patoplástico, la forma o el contenido?

Con el modelo patogenia/patoplastia se intenta confirmar la universalidad de los crite- rios diagnósticos a costa de reducir la importancia del factor local. La dimensión cultural queda así limitada a los aspectos epifenoménicos de los trastornos mentales; esto es, a si el delirante se cree un espía de la CIA o del Mossad. Sin embargo, la argumentación que permite llegar a esta deducción no se basa en criterios de objetividad, pues la forma no es siempre invariable, ni el contenido debe entenderse como una realidad secundaria, ya que al fin y al cabo es la que organiza la experiencia del paciente. Una prueba adicional a nuestra argumentación es que el factor cultural desborda el plano sintomatológico para afectar otras variables clínicas como el pronóstico.

\section{El dilema de la diferencia en el pronóstico de enfermedades entre los países desarrollados y los países en vías de desarrollo}

Las investigaciones transculturales nos informan de un mejor pronóstico de la esquizofrenia en los países en vías de desarrollo en comparación con los países desarrollados 38. Las hipótesis para explicar esta variabilidad son múltiples. La propia Organización Mundial de la Salud (OMS) indicaba en el IPSS algunas pistas que podrían guiar los estudios posteriores, como la posibilidad de que estuvieran involucrados factores locales (estructura familiar, factores económicos), que la variabilidad en el pronóstico se debiera a diferencias de tratamiento o que los síntomas visibles por el profesional respondieran en el fondo a enfermedades diferentes.

En el DOSMD la hipótesis cultural cobra más fuerza, ya que los resultados replican la variabilidad en el pronóstico y el diseño de cohortes, según inicio agudo o insidioso de esta investigación permite descartar un posible sesgo de muestreo, por el cual en los países en vías de desarrollo se estuviesen incluyendo más pacientes con inicio agudo, y por tanto mejor pronóstico, que en los países desarrollados. Por otro lado, la hipótesis de los factores socioculturales parece congruente con estudios anteriores, como los de Waxler 39 , quien a partir de sus trabajos comparativos había planteado que el pronóstico dependía de la respuesta social.

La idea de Waxler deriva de su observación de las familias cingalesas, las cuales presentan ante un caso de esquizofrenia un curioso comportamiento, pues no aceptan ni un diagnóstico de incurabilidad para el afectado ni la posibilidad de una cronificación, sino que negocian el diagnóstico con el profesional, ya sea psiquiatra o profesional folk. El motivo es que no conciben que el individuo sea responsable 
de su enfermedad, sino que su cuerpo o alma están poseídos. De esta manera, las expectativas sociales sobre el curso de la enfermedad se convierten en beneficiosas, ya que se espera que una vez recuperado de la posesión el afectado volverá a su estado normal.

Si bien esta idea ha sido discutida 40 , la hipótesis sociocultural ha generado un interesante campo de investigación para epidemiólogos y etnógrafos 41,42 . Hoy por hoy podemos hablar de cuatro líneas de estudio que, si bien no son excluyentes ni contradictorias entre sí, pueden descomponerse a efectos analíticos.

La primera línea ha enfatizado el papel del trabajo y de las variables económico-políticas 43,44 . Concretamente se alude a que la estructura de empleo formalizado de las sociedades de capitalismo avanzado junto a la demanda de habilidades a los trabajadores se convierten en obstáculos a la reinserción social de los pacientes, al menos en comparación con el sistema de tareas no formalizadas que caracteriza otros contextos como los rurales. Adicionalmente, parece existir una asociación entre crisis económicas, altas tasas de desempleo y mayor número de ingresos y re-ingresos en los hospitales psiquiátricos cuando se analizan los datos desde una perspectiva histórica. La dificultad de acceso a un empleo adquiere en este punto capacidad explicativa para entender las diferencias de pronóstico a partir de la mayor o menor reinserción de los afectados.

El impacto de las concepciones culturales constituye una segunda línea de investigación relevante. Las ideas de persona, la manera de entender la enfermedad, las expectativas de curación o cronicidad son, de hecho, factores fundamentales para el pronóstico. Por ejemplo, en un estudio 45 se compararon las concepciones de la psicosis entre familias hispanas, africanoamericanas y euro-americanas en Estados Unidos. Los resultados son reveladores: mientras el $65 \%$ de las primeras pensaban que la enfermedad tenía curación, sólo el 19\% de las familias euro-americanas concebían esta posibilidad. Inversamente, mientras sólo el $31 \%$ de los hispanos entendían la enfermedad mental como incurable, el $81 \%$ de los euro-americanos afirmaban que los trastornos mentales severos eran incurables Un interesante resultado que podría explicar el mejor pronóstico de la esquizofrenia en algunos colectivos hispanos.

El tercer tema investigado ha sido el impacto de la familia a partir de variables como la Emoción Expresada; un término y una técnica para valorar las actitudes emocionales de los familiares hacia los pacientes. Es sabido que a mayor Emoción Expresada, principalmente en sus dimensiones de hostilidad y criticismo, se producen más recaídas, peor curso de la enfermedad, menor inserción psicosocial y más sintomatología. Las actitudes de la familia, los procesos de estigmatización, el tipo de estructura de parentesco y el papel de soporte o de criticismo de ésta son, de hecho, variables que resultan alumbradoras de las diferencias de pronóstico en las investigaciones comparativas 46,47 .

En último lugar tenemos los estudios sobre el impacto del tamaño y morfología de las redes sociales 48 . Se trata de una variable profusamente estudiada en relación con las rehospitalizaciones, la calidad de vida y el desarrollo de habilidades. En síntesis podemos decir que las redes caracterizadas por una alta densidad es decir, aquéllas en donde la mayor parte de los actores que envuelven a ego interactúan entre sí - parecen tener un efecto protector en cuanto a las recaídas en los contextos culturales de baja estigmatización. Contrariamente, la densidad de la red se convierte en un predictor de mal pronóstico en contextos urbano-industriales, cuando el marco de concepciones culturales supone la exclusión de los enfermos. En realidad, las redes más adecuadas siempre son aquellas que replican la estructura estándar del contexto: las redes de alta densidad en sistemas sociales de pequeña escala o Gemeinschaft y las redes abiertas y dispersas en sistemas urbano-industriales o Gesellschaft.

\section{Los problemas en la traducción}

\section{y adaptación cultural de los instrumentos diagnósticos y las escalas}

Otro de los focos actuales de investigación es la adaptación de los instrumentos diagnósticos y epidemiológicos. Desde un punto de vista psicométrico, la traducción de un cuestionario constituye un proceso técnicamente complejo para el que existen procedimientos ya protocolizados. Sin embargo, y a pesar de este esfuerzo técnico, la cuestión de la fiabilidad y validez de los instrumentos ha levantado enormes suspicacias 49,50. Una de las causas es que desde la epidemiología se tiende a pensar que una simple traducción lingüística es suficiente. Pero y éste es el problema - cada cultura muestra patrones diferenciados de expresión de la aflicción y de la enfermedad. La cultura no es sólo un esquema cognitivo de representaciones, símbolos y formas de pensar, sino también un sistema que modela la experiencia y emocionalidad humanas. La idea de culpa, por ejemplo, adquiere poco sentido en determinadas culturas que enfatizan la vergüenza y/o la deshonra 51. Los contenidos que propician una si- 
tuación de malestar varían también a lo largo de las diferentes sociedades. Como resultado, las pretensiones de homogeneidad se tornan precarias, porque los instrumentos se enfrentan a realidades autóctonas poco compatibles con los criterios diagnósticos.

Obeyesekere 52 ha planteado el problema con ironía mediante un ejercicio de inversión etnocéntrica. Supongamos, nos dice, que tomamos a un varón o mujer sudasiático que presenta los siguientes síntomas: pérdida brusca de peso, fantasías sexuales, poluciones o emisiones nocturnas y decoloración de la orina. En el Sur de Asia el paciente será diagnosticado de forma indefectible como un caso de "pérdida de semen" (un culture-bound syndrome conocido como dhat), aunque en términos operacionales podamos encontrar esta constelación de síntomas en muchas sociedades, desde China a Perú. Imaginemos por un momento que elaboramos un instrumento diagnóstico siguiendo la sintomatología apuntada y que lo utilizamos entre la población norteamericana. Con toda seguridad encontraremos una tasa considerable de prevalencia de dhat, con lo cual podremos concluir que estamos ante un síndrome universal.

El dilema de Obeyesekere pone en cuestión los procedimientos metodológicos utilizados en los estudios de prevalencia y, a la vez, visibiliza el problema de la falacia categorial que antes hemos analizado. Con todo, lo que queremos resaltar aquí es que la adaptación de los instrumentos diagnósticos requiere de una traducción no sólo idiomática, sino también cultural. Los mundos locales no son contextos epifenoménicos que puedan soslayarse mediante artificios metodológicos, sino realidades que constituyen una de las fuentes principales de la variación en psicopatología. El contexto cultural no puede continuar siendo el eslabón perdido u olvidado de las investigaciones en psiquiatría.

\section{La asistencia, o el reto de la multiculturalidad}

Si bien la diversidad étnica y cultural es un rasgo distintivo de las sociedades contemporáneas, la multiculturalidad no es un fenómeno nuevo. Como indica Lévi-Strauss 53, las sociedades humanas nunca han estado solas, ya que siempre han dependido de un otro real o ficticio para construir su identidad. El elemento que marca la diferencia en la modernidad no es por tanto la diversidad, sino el grado de esta diversidad y los procesos de hibridación. El in- cremento de las migraciones transnacionales ha producido un efecto hasta ahora desconocido de desplazamiento y desubicación de los grupos humanos que precisa de la formulación de nuevas políticas de cohesión social y convivencia. Las tecnologías de la información han producido un efecto de empequeñecimiento del mundo y un aumento de la comunicación entre lugares distantes que ha permitido la modificación de las coordenadas espacio-temporales 54: ahora lo simultáneo en el tiempo no es necesariamente contiguo en el espacio. Los procesos de globalización económica han generado una mayor interdependencia regional y un mayor desequilibrio en la distribución de los recursos que tiene efectos sobre los sistemas sanitarios: ausencia de sistemas de atención en salud mental en muchos países pobres, dificultad de acceso a los tratamientos, inequidades en la atención, etc. La cultura de la modernidad, por su parte, ha adquirido el carácter de metacultura que filtra en los diferentes mundos locales y que incluso mediatiza nuestro contacto con los productos y representaciones de estos mundos a partir de la relación consumidormercancía. Tallas senegalesas, comida china, noticias sobre los movimientos indígenas en México, música popular europea, son productos culturales desprendidos de su mundo local que han sido incluidos en sistemas globalizados de mercantilización y consumo. El mundo en el que vivimos es cada vez más un espacio de culturas líquidas y de hibridación desigual entre la metacultura global y los diferentes mundos locales. Incluso los movimientos de defensa de la identidad pueden entenderse como formas de resistencia a este proceso de hibridación desigual que a la vez que lo contestan permiten reproducirlo.

En este escenario global, los servicios de salud mental deben afrontar el reto de la interculturalidad. Los culture-bound síndromes que se circunscribían a contextos foráneos conforman ahora la cotidianeidad de la asistencia psiquiátrica. Lo mismo podemos decir de la diversidad sintomatológica o de la mayor presencia de trastornos adaptativos y de síndromes por estrés postraumático como consecuencia de los desarreglos de la globalización 55 : conflictos bélicos, migraciones, desplazamientos de población, pobreza, etc. Los problemas derivados de la exclusión, la marginación y el racismo también adquieren relevancia en la salud mental, pues los problemas estructurales se materializan en la vida de personas concretas en forma de frustración, baja auto-estima, agresividad, violencia y otras emociones y comportamientos. 
Las respuestas a estas cuestiones han sido diversas en los diferentes contextos nacionales, pues la implementación de políticas públicas en salud mental ha sido dependiente de los modelos de ciudadanía más amplios que han englobado y ofrecido sentido a estas iniciativas. La reducción de las desigualdades en el acceso al sistema sanitario, la sensibilidad hacia las identidades y particularidades en el contexto de una ciudadanía multicultural o la simple negación de la diversidad han sido algunas de estas respuestas.

El modelo británico 56, por ejemplo, ha tomado como base un liberalismo clásico asociado con su historia colonial y ha entendido que la respuesta más adecuada era la lucha antirracista en el acceso a los servicios sanitarios. Sin embargo, sus iniciativas están lejos de cumplir el ideal liberal de disminuir la brecha entre ciudadanos de primera y ciudadanos de segunda. El republicanismo francés 57 , con la delegación de la identidad cultural y étnica en el individuo, ha soslayado las desigualdades sociales que se imbrican con las identidades culturales y que afectan finalmente al ejercicio real de los derechos de ciudadanía. El modelo multicultural (liberalismo multicultural) de Canadá 58 no ha podido resolver el riesgo de que ante determinadas situaciones de desigualdad el reconocimiento de los derechos colectivos puede llevar a la conformación de guetos que favorece la segregación y la injusticia social. Las políticas estadounidenses, por su parte, han tendido a retificar la noción de etnicidad, y lo que es más preocupante, incluso de raza, generando un modelo de "competencia cultural" 59 que puede resultar tan formalizado como amplificador de las diferencias, a pesar de su considerable predicamento en la literatura médica reciente.

La competencia cultural se ha definido 59 como el conjunto de habilidades académicas e interpersonales que permiten que los individuos incrementen su comprensión y apreciación de las diferencias y similitudes culturales de los grupos humanos. La definición parece en principio adecuada. El problema se encuentra en su praxis. ¿Cómo congeniar un modelo basado en la observación, característico del neokraepelinismo y las tendencias biológicas, con la escucha atenta necesaria para la sensibilidad cultural? ¿Dónde se ubica la cultura profesional en este escenario? ¿Qué debemos entender como cultura, el equipaje simbólico de los pacientes de grupos no mayoritarios o cualquier mundo de representaciones y prácticas de los usuarios? Estas cuestiones no han recibido una respuesta adecuada desde el modelo de competencia cultural.
En realidad, la aplicación de la noción de competencia cultural ha presentado diversos riesgos y deficiencias en su puesta en práctica. Sin ánimo de ser exhaustivos, algunos de ellos han sido:

1) El énfasis en las habilidades de los proveedores de salud, en detrimento del análisis del sistema sanitario y del escenario social más amplio;

2) La tendencia a cosificar las referencias culturales de los usuarios mediante un tratamiento asistemático de este tipo de variables en las historias clínicas. Por ejemplo, a menudo se ha comprendido la identidad étnica del paciente como una información del mismo orden que el diagnóstico, el tratamiento o la sintomatología. Este error radica en la falta de formación de los profesionales de la salud mental en antropología y en ciencias sociales que lleva a tratar los factores socioculturales como variables biológicas;

3) La escasa atención prestada a las condiciones materiales asociadas con la diversidad cultural, especialmente en los colectivos más vulnerables (inmigrantes ilegales, refugiados, etc.); 4) La comprensión de la cultura como un conjunto de creencias más que como un sistema que organiza la experiencia subjetiva. La noción de creencia deviene, finalmente, en una minusvaloración de los saberes populares y sus recursos terapéuticos;

5) La reducción de la cultura al ámbito de la comunicación clínica, cuando también constituye un contexto explicativo de la etiología, la sintomatología, el curso, evolución y tratamiento de los trastornos mentales;

6) La atribución de la "cultura" exclusivamente a los usuarios pertenecientes a las minorías étnicas, de tal forma que los pacientes de los grupos mayoritarios se convierten en sujetos "aculturales", así como los propios profesionales; 7) La concepción de la cultura como una realidad estable, cuando la modernidad se caracteriza por el dinamismo y la hibridación culturales; 8) La ausencia de principios metodológicos para investigar la asociación entre cultura y poder, principalmente cuando estamos hablando de grupos sociales caracterizados por la exclusión, la marginación y el racismo;

9) La excesiva protocolización de los procedimientos, que impide una contextualización, valoración y tratamiento adaptados a la especificidad de cada caso;

10) La creación de estereotipos culturales y/o étnicos que no dan cuenta de la diversidad interna existente en toda cultura y que pueden correr el riesgo de enquistarse en la forma de prejuicios. 
Una adecuada competencia cultural de los profesionales debe ir más allá del simple conocimiento y reproducción de estereotipos como si fuesen rasgos clínicos. Entre otras cosas porque la cultura es una realidad dinámica que se presta a la diversidad interna, a los pidgins y al mestizaje. La reflexión sobre la propia cultura profesional, la cultura local y los efectos de la globalización es el punto de partida para una actitud de competencia en este ámbito, porque mueve a un diálogo entre saberes y presunciones. La capacidad de situarse "entre" un mundo profesional conocido y un mundo por conocer es el criterio que a nuestro juicio debe definir la competencia (inter)cultural en un escenario en donde lo exótico es ya lo cotidiano.

\section{Conclusión}

En este artículo hemos reflexionado críticamente sobre tres ámbitos de la psiquiatría cultural: el descubrimiento de la cultura profesional, la investigación de la variabilidad de los cuadros clínicos y la adecuación de los servicios de salud mental a la realidad multicultural de nuestro tiempo. Por razones de extensión, hemos prescindido de otros temas que también deberían recibir atención, como la eficacia simbólica de las terapias folk, el análisis del sueño o la etnopsicofarmacología.

El descubrimiento de la cultura profesional ha supuesto hacer consciente que la psiquiatría, como cualquier campo científico-técnico, es un sistema de y para la realidad. Esto es: un sistema que permite dar significado (nosologías) al paisaje psicopatológico y a la vez modificarlo mediante determinadas incursiones empíricas (tratamientos). La conjunción entre el mundo de representaciones en el que ha sido socializado el profesional y la dimensión empírico-práctica favorece la ilusión de un mundo estable de presunciones, principalmente cuando se "naturaliza" el propio sistema cultural convirtiéndolo en la representación de la realidad más que en una representación de la realidad. Esta ilusión obstaculiza la posibilidad de un conocimiento racional y verdaderamente científico de los trastornos mentales, pues parafraseando a Bourdieu 60 podemos decir que el progreso del conocimiento es indisociable del progreso en el conocimiento de las condiciones del conocimiento.

El problema de la variabilidad, por su parte, ha suscitado diferentes problemas teóricos y metodológicos, entre los que cabe destacar el uso - o casi sería mejor decir el rescate - de fórmulas ad hoc creadas por la psiquiatría del siglo XIX, como el modelo patogenia/patoplastia. Atribuir toda variación a la patoplastia y toda uniformidad a la patogenia recuerda el viejo dicho de disparar la flecha y después pintar la diana. Es necesario que las investigaciones intenten dar cuenta de la diversidad de manifestaciones, incluso más allá de los criterios diagnósticos definidos a priori, para dibujar el escenario de variación de los trastornos mentales. Para ello puede ser útil la conjunción de las grandes investigaciones epidemiológicas - con sus sofisticados instrumentos y protocolos de medición - con el análisis más microscópico y cualitativo de los mundos locales donde los afectados viven e interactúan.

En última instancia, el reto de la asistencia en contextos multiculturales nos dirige al territorio de las políticas públicas en salud mental; un ámbito que no puede prescindir del análisis de la propia cultura profesional. Cuando este análisis no está presente, la trivialización de la cultura del paciente mediante su reducción a problemas de comunicación y su inserción en protocolos preestablecidos puede inducir a la confusión entre una "psiquiatría cultural" y una "psiquiatría de la cultura". Y es muy diferente el análisis cultural del paciente que la patologización de sus rasgos étnicos. La "competencia cultural" debe dejar paso a una "competencia intercultural" que nos permita vislumbrar la atención como un diálogo entre interpretaciones de la realidad, entre el clínico y el paciente, entre el conocimiento psiquiátrico y las representaciones locales, entre la cultura profesional y la cultura local. Sólo así podremos enfrentarnos a un mundo intercultural. 


\section{Resumen}

La cultura influye en la experiencia, la expresión, el curso, la evolución y el pronóstico de los trastornos mentales, así como en las terapias y las políticas de salud mental. No obstante, la cultura ha sido una dimensión negada en la psiquiatría general, que no ha integrado apropiadamente este factor en la investigación epidemiológica, la práctica clínica y las políticas sanitarias. Este artículo intenta contribuir al debate teórico en la psiquiatría cultural mediante una revisión de: (i) las discusiones teóricas y los estudios etnográficos sobre los dispositivos psiquiátricos, (ii) las investigaciones transculturales comparativas de los trastornos mentales y (iii) el uso del modelo de "competencia cultural" con las minorías étnicas y los refugiados.

Salud Mental; Psiquiatría; Cultura

\section{Referências}

1. Ebigbo PO. Development of a culture specific (Nigeria) screening scale of somatic complaints indicating psychiatric distress. Cult Med Psychiatry $1982 ; 6: 29-44$.

2. Kirmayer LJ, Minas H. The future of cultural psychiatry: an international perspective. Can J Psychiatry 2000; 45:438-46.

3. Wittkower ED, Prince RH. A review of transcultural psychiatry. In: Caplan G, editor. American handbook of psychiatry. v. 2. 2nd Ed. New York: Basic Books; 1974. p. 535-50.

4. Devereux G. Essais d'ethnopsychiatrie genérale. Paris: Gallimard; 1970.

5. Guyotat J. Estudios de antropología psiquiátrica. México DF: Fondo de Cultura Económica; 1994.

6. Murphy HBM. Comparative psychiatry: the international and intercultural distribution of mental illness. New York: Springer-Verlag; 1982.

7. De Jong JT; van Ommeren M. Mental health services in a multicultural society: interculturalization and its quality surveillance. Transcult Psychiatry 2005 ; 42:437-56.

8. Tseng WS. Handbook of cultural psychiatry. San Diego: Academic Press; 2001.

9. Lewis-Fernandez R, Kleinman A. Cultural psychiatry. Theoretical, clinical, and research issues. Psychiatr Clin North Am 1995; 18:433-48.

10. Kraepelin E. Psychiatrie. Ein Lehrbuch für Studierende und Ärzte. 8th Ed. Leipzig: Verlag Von Johann Ambrousius Barth; 1909.

11. Kiev A. Mexican-American folk psychiatry. New York/London: The Free Press; 1968.
12. Devereux G. Normal and abnormal. In: Casagrande J, Glad T, editors. Some uses of anthropology. Theoretical and applied. Washington DC: Anthropological Society of Washington; 1956. p. 11-135.

13. Róheim G. The gates of the dream. New York: International Universities Press Inc.; 1979.

14. Kiev A, editor. Magic, faith and healing: studies in primitive psychiatry today. New York: The Free Press; 1974.

15. Ortigues MC, Ortigues E. L'Oedipe Africain. Paris: Plon; 1966.

16. Bibeau G. Cultural psychiatry in a creolizing world. Questions for a new research agenda. Transcult Psychiatry 1997; 34:9-41.

17. Littlewood R, Simone D, editors. Cultural psychiatry and medical anthropology: an introduction and reader. London: Athlon Press; 2000.

18. American Psychiatric Association. Diagnostical and statistical manual of mental disorders. 4th Ed. Washington DC: American Psychiatric Association; 1994.

19. Kleinman A. Rethinking psychiatry: from cultural category to personal experience. New York: The Free Press; 1988.

20. Mezzich JE, Kirmayer LJ, Kleinman A, Fabrega Jr. H, Parron DL, Good BJ, et al. The place of culture in DSM-IV. J Nerv Ment Dis 1999; 187:457-64.

21. Young A. Emil Kraepelin and the origins of American psychiatric diagnosis. Curare 1991; Special Volume 7:175-81.

22. Young A. The harmony of illusions. An ethnographic account of posttraumatic stress disorder. Princeton: Princeton University Press; 1995. 
23. World Health Organization. Schizophrenia: an international follow-up study. New York: Wiley; 1979.

24. Breuer J, Freud S. Estudios sobre la histeria. Buenos Aires: Amorrortu Editores; 1895.

25. American Psychiatric Association. Diagnostical and statistical manual of mental disorders. $4^{\text {th }} \mathrm{Re}$ vised Ed. Washington DC: American Psychiatric Association; 2000.

26. American Psychiatric Association. Diagnostical and statistical manual of mental disorders. 3rd Ed. Washington DC: American Psychiatric Association; 1980.

27. American Psychiatric Association. Diagnostical and statistical manual of mental disorders. $3^{\text {rd }}$ Revised Ed. Washington DC: American Psychiatric Association; 1987.

28. Martínez-Hernáez A. What's behind the symptom? Amsterdam: Harwood Academic Publishers; 2000.

29. Kleinman AM. Depression, somatization and the "new cross-cultural psychiatry". Soc Sci Med 1977; 11:3-10.

30. Goffman E. The insanity of place. Psychiatry 1969; 32:357-88.

31. Jablensky A, Sartorius N, Ernberg G, Anker M, Korten A, Cooper JE, et al. Schizophrenia: manifestations, incidence and course in different cultures. A World Health Organization ten-country study. Psychol Med Monogr Suppl 1992; 20:1-97.

32. Sartorius N, Jablensky A, Gulbinat W, Ernberg G. WHO collaborative study: assessment of depressive disorders. Psychol Med 1980; 10:743-9.

33. World Health Organization. The World Mental Health Survey Initiative. http://www.hcp.med. harvard.edu/wmh/index.php (accedido el 07/Dic/ 2005).

34. Chen H, Guarnaccia PJ, Chung H. Self-attention as a mediator of cultural influences on depression. Int J Soc Psychiatry 2003; 49:192-203.

35. Bhugra D, Mastrogianni A. Globalization and mental disorders. Overview with relation to depression. Br J Psychiatry 2004; 184:10-20.

36. Parsons CD, Wakeley P. Idioms of distress: somatic responses to distress in everyday life. Cult Med Psychiatry 1991; 15:111-32.

37. Leff J. Psychiatry around the globe. A transcultural view. 2nd Ed. Plymouth: Gaskell; 1988.

38. Sartorius N, Gulbinat W, Harrison G, Laska E, Siegel C. Long-term follow-up of schizophrenia in 16 countries. A description of the International Study of Schizophrenia conducted by the World Health Organization. Soc Psychiatry Psychiatr Epidemiol 1996; 31:249-58.

39. Waxler NE. Is outcome for schizophrenia better in non-industrial societies? The case of Sri Lanka. J Nerv Ment Dis 1979; 167:144-58.

40. Cohen A. Prognosis for schizophrenia in the Third World: a reevaluation of cross-cultural research. Cult Med Psychiatry 1992; 16:53-75.

41. Thara R. Twenty-year course of schizophrenia: the Madras Longitudinal Study. Can J Psychiatry 2004; 49:564-9.

42. Halliburton M. Finding a fit: psychiatric pluralism in south India and its implications for WHO studies of mental disorder. Transcult Psychiatry 2004; 41:80-98.
43. Warner R. Recovery from schizophrenia: psychiatry and political economy. London: Routledge/ Kegan Paul; 1985.

44. Warner R. Commentary on Cohen, Prognosis for schizophrenia in the Third World. Cult Med Psychiatry 1992; 16:85-8.

45. Guarnaccia P, Parra P, Deschamps A, Milstein G Argiles N. Si Dios quiere: Hispanic families' experiences of caring for a seriously mentally ill family member. Cult Med Psychiatry 1992; 16:187-215.

46. Karno M, Jenkins JH, De La Selva A, Santana F Telles C, Lopez S. et al. Expressed Emotion and schizophrenia outcome among Mexican-American families. J Nerv Ment Dis 1987; 175:143-51.

47. Wig NN, Menon DK, Bedi H, Leff J, Kuipers L, Ghosh A, et al. Expressed emotion and schizophrenia in north India. II. Distribution of expressed emotion components among relatives of schizophrenic patients in Aarhus and Chandigarh. Br J Psychiatry 1987; 151:160-5.

48. Martínez-Hernáez A. El pronóstico de la esquizofrenia. Un enigma común a la psiquiatría y a las ciencias sociales. Arch Neurobiol 1999; 62: 135-50.

49. Canino G, Lewis-Fernandez R, Bravo M. Methodological challenges in cross-cultural mental health research. Transcult Psychiatry 1997; 34:163-84.

50. Bhui K, Bhugra D. Transcultural psychiatry: some social and epidemiological research issues. Int J Soc Psychiatry 2001; 47:1-9.

51. Kinzie JD, Manson SM, Vinh DT, Tolan NT, Anh B, Pho TN. Development and validation of a Vietnamese-language depression rating scale. Am J Psychiatry 1982; 139:1276-81.

52. Obeyesekere G. Depression, buddhism, and the work of culture in Sri Lanka. In: Kleinman AM, Good BJ, editors. Culture and depression. Studies in the anthropology and cross-cultural psychiatry of affect and disorder. Berkeley: University of California Press; 1985. p. 134-52.

53. Lévi-Strauss C. Raza y cultura. Madrid: Cátedra; 1996.

54. Castells M. La era de la información. Economía sociedad y cultura. Madrid: Alianza Editorial; 1998.

55. Desjarlais R, Eisenberg L, Good B, Kleinman AM World mental health: problems and priorities in low-income countries. New York: Oxford University Press; 1995.

56. Littlewood R. Ideology, camouflage or contingency? Racism in British psychiatry. Transcult Psychiatry 1993; 30:243-91.

57. Freeman P. Ethnopsychiatry in France. Transcult Psychiatry 1997; 34:313-9.

58. Kymlicka W. Multicultural citizenship. Oxford: Oxford University Press; 1995.

59. US Department of Health and Human Services. Mental health: culture, race and ethnicity - a supplement to the mental health report of the surgeon general. Rockville: US Department of Health and Human Services; 1991.

60. Bourdieu P. Le sens pratique. Paris: Editorial Minuit; 1980.

Recibido el 27/Dic/2005

Aprobado el 15/Ago/2006 